\title{
Notch Signaling in the Regulation of Hematopoietic Stem Cell
}

\author{
Fabio Pereira Lampreia ${ }^{1}$. Joana Gonçalves Carmelo ${ }^{1,2} \cdot$ Fernando Anjos-Afonso $^{1}$
}

Published online: 10 July 2017

(C) The Author(s) 2017. This article is an open access publication

\begin{abstract}
Purpose of Review Understanding the signaling pathways that govern hematopoietic stem and progenitor cells (HSPCs) is fundamental to uncover their regulation and how this is skewed in hematological malignancies. Whether Notch is necessary for the regulation of mammalian HSPCs is still unclear. We therefore critically review the current literature on the role of Notch in HSPCs.

Recent Findings HSPCs have shown different requirements for Notch signals in vitro and in vivo and at different stages of differentiation. Additionally, bone marrow niche cells activate Notch signaling in HSPCs enhancing their regenerative and self-renewal capacity.

Summary Despite the controversy, adequate levels of Notch signaling appear necessary to avoid the development of hematological malignancies. Contrary to early studies, recent
\end{abstract}

Fabio Pereira Lampreia and Joana Gonçalves Carmelo are equal contributors of this work.

This article is part of the Topical Collection on Cell:Cell Interactions in Stem Cell Maintenance

Fernando Anjos-Afonso

dosanjosafonsof@ cardiff.ac.uk

Fabio Pereira Lampreia

LampreiaFG@ cardiff.ac.uk

Joana Gonçalves Carmelo

carmelogoncalvesJI@ cardiff.ac.uk

1 Haematopoietic Signalling Group, European Cancer Stem Cell Research Institute, School of Biosciences, Cardiff University, Hadyn Ellis Building, Maindy Road, Cardiff CF24 4HQ, UK

2 Department of Bioengineering and iBB-Institute for Bioengineering and Biosciences, Instituto Superior Técnico, Universidade de Lisboa, Av. Rovisco Pais, 1049-001 Lisbon, Portugal research suggests that Notch signaling may play a role in homeostatic and regenerative hematopoiesis but further investigation is necessary to understand how it is regulated by the different ligand/receptor pairings and the molecular mechanisms that are triggered.

Keywords Notch signaling $\cdot \mathrm{HSC} \cdot \mathrm{Niche} \cdot \mathrm{MPD}$

\section{Introduction: Brief Description of Notch Signaling}

Detailed reviews on the Notch pathway can be found elsewhere [1, 2]. Briefly, in mammals, there are four Notch receptors (Notch1, 2, 3, and 4) and five structurally related, singlepass membrane Notch ligands (Delta-like1, 3, and 4 and Jagged1 and 2). The canonical Notch signaling is activated through ligand-mediated interaction that instigates the first cleavage of the receptor, which is mediated by metalloproteases like the tumor necrosis factor alphaconverting enzyme (TACE). This licenses a second cleavage mediated by the $\gamma$-secretase complex (comprising Nicastrin, Presenilin 1/2, PEN2 (Presenilin enhancer 2), and APH1 (anterior pharynx-defective 1)) that forms the intracellular Notch receptor domain (N-ICD), which subsequently translocates into the nucleus and cooperates with the DNA-binding protein RBPJK (recombinant binding protein suppressor of hairless; also known as CSL). This allows the conversion of the transcriptional repressor complex into an activator complex together with its co-activator Mastermind-like 1 (MAML1), and other specific co-activators $[1,2]$. The activation of Notch signaling triggers the expression of various target genes, such as Hes and the Hes-related (HESR/HEY) family of basic helix-loop-helix transcription repressors [3] and subsequently regulates the expression of other genes. There is a growing appreciation that this pathway can also be 
orchestrated in a non-canonical manner, via HES- or RBPJkHES-independent axis or by other means [4].

\section{First Evidences of Notch Signaling in the Regulation of HSPC Biology}

The blood is one of the most highly regenerative tissues with an average of $10^{11}-10^{12}$ new blood cells being produced daily in a healthy adult human. With such a high turnover system, a very fine-tuned production of cells is required to maintain the homeostasis of the blood system. Studies on signaling pathways that govern hematopoietic stem cell (HSC) self-renewal and fate decisions are critical for this balance and some of the HSC regulators have been identified including cytokines, growth factors, transcription factors, cell cycle regulators, chromatin modifiers, etc. However, the molecular mechanisms in particular the cell-to-cell interactions that support and regulate HSCs in their microenvironment (niche) are still largely unexplored $[5,6]$. The evolutionarily conserved Notch signaling pathway functions as a major mediator of cell fate determination during development and also regulates diverse cellular functions including differentiation, proliferation, and survival. In hematopoiesis, the importance of Notch signaling has been associated with hematological malignancies such as T cell acute lymphoblastic leukemia [7, 8]. Differently from a conventional signaling pathway that involves the interaction between soluble factors and their receptors, Notch signaling pathway has both ligand and receptors as transmembrane proteins expressed on the cell surface. As such, Notch signaling is in many ways an ideal candidate pathway for instructing communication between HSCs and their niche, as it requires cellto-cell contact for activation.

While known to be required during the development of the hematopoietic system [9] and in T cell development [10], whether Notch signaling plays a role in the regulation of adult hematopoietic stem cells in physiological conditions remains a subject of active debate mainly due to conflicting results obtained in several studies. First, Notch ligands and receptors have been detected in the bone marrow niche [11] and in HSPCs, respectively, both in mice and humans [12, 13]. Then, when transducing the constitutively active form of Notch1-intracellular domain (N1-ICD) [14] or the Notch target Hes 1 [15] results is an increase in the self-renewal capacity of HSPCs. Further to this, activation of Notch signaling through exposure of primitive hematopoietic cells to Notch ligands in vitro such as Jagged1 [11], extracellular Deltalike1 ligand [16], and soluble Jagged1 [17] also promotes stem cell self-renewal and inhibits differentiation.

Based on these early reports, it is therefore tempting to suggest that Notch signaling might play a role in hematopoiesis. Nonetheless, this effect is likely to be the result of superphysiological levels of Notch signaling in vitro. On the other hand, in an interesting study, it was reported that the loss of Itch (an E3 ubiquitin ligase which negatively regulates Notch signaling by controlling Notch receptor degradation) led to sustained levels of Notch1-mediated signaling, thus, functioning as an indirect Notch gain-of-function approach [18]. As a result, mice that were transplanted with Itch-deficient $\left(\mathrm{Lin}^{-} \mathrm{Sca}^{+}{ }^{+} \mathrm{Kit}^{+}\right)$LSK HSPCs had an expanded stem cell pool with enhanced hematopoietic contribution up to 24 weeks as compared to control LSK cells.

Despite these positive results that paved the way for approaches that allowed the development of in vitro HSPCs expansion protocols [19, 20], the understanding of the functional role of Notch signaling remains unclear as loss of function approaches have led to the proposal that the Notch pathway is not required in steady state hematopoiesis, as highlighted below (Table 1), in contrast to the in vitro studies.

While early loss of mouse Notch1 [10] and Rbpj [21] studies have established the Notch1-RBPJk-HES1 axis as a major regulator of $\mathrm{T}$ cell differentiation, no myeloid and $\mathrm{B}$ cell lineage defects were observed. Although these studies did not focus on HSCs, Duncan and colleagues showed that LSK cells from a transgenic Notch reporter (TNR-GFP) mouse have high Notch activity as evidenced in bone marrow sections by co-localization of c-kit ${ }^{+}$and $\mathrm{GFP}^{+}$cells and by flow cytometric analysis of primitive LSK cells showing GFP expression. Interestingly, transplanting murine lineage negative ( Lin $^{-}$) cells transduced with dominant-negative of Xenopus suppressor of Xenopus Rbpj homologue $(\mathrm{dnXSu}(\mathrm{H}))$ resulted in the depletion of the HSC population after long-term reconstitution compared to control transduced cells [12].

Which Notch ligand/receptor pairing is preferentially used by HSPCs to signal Notch still remains elusive. Jagged1 is expressed in the bone marrow niche cells, in particular on osteoblasts [11, 27••]. Yet, loss of both Jagged1 and Notch1 in the bone marrow had no effect on HSC homeostasis, on hematopoietic reconstitution after genotoxic insult, or in the number of cells of different stem and progenitor subsets after bone marrow transplant thus excluding an essential role for Jagged1-mediated Notch1 signaling on HSC self-renewal or differentiation [22]. However, these studies did not rule out the possible redundant effects from other Notch receptors or ligands. Indeed, Varnum-Finney and colleagues have demonstrated that Notch2 but not Notch1 mediates LSK cell selfrenewal, repopulation following transplantation and inhibits myeloid differentiation. Yet, neither loss of Notch1 nor Notch2 had any effect on the number of HSCs in steadystate conditions [24].

Perhaps more convincingly is a study undertaking a paninhibition of all canonical Notch signaling approach mediated by the overexpression of a dominant negative form of Mastermind-like 1 (dnMAML1). Using this approach, no effect on LSK cell function was reported with comparable repopulation capacities as to control [23]. The authors have 
Table 1 Evidences of Notch signaling in HSPC regulation

\begin{tabular}{|c|c|c|c|c|c|}
\hline Study & Method & Species & Effect on general hematopoiesis & Effect on HSCs/HSPCs & Reference \\
\hline Notch1 loss of function & Mx-Cre & Mouse & $\begin{array}{l}\text { Impaired T cell development; } \\
\text { myeloid and B cell } \\
\text { development is normal }\end{array}$ & ND & {$[10]$} \\
\hline RBPJk loss of function & Mx-Cre & Mouse & $\begin{array}{l}\text { Impaired T cell development; } \\
\text { myeloid and B cell } \\
\text { development is normal }\end{array}$ & ND & {$[21]$} \\
\hline $\begin{array}{l}\text { Notch inhibition (dnRBPJk } \\
\text { transduced LSK cells) }\end{array}$ & $\begin{array}{l}\text { Transduction LSK cells } \\
\text { with } \operatorname{dnMAML1} \\
\text { or } \mathrm{dnXSu}(\mathrm{H})\end{array}$ & Mouse & $\begin{array}{l}\text { Accelerated differentiation } \\
\text { towards B and myeloid } \\
\text { cell lineages }\end{array}$ & Depletion of LT-HSC & [12] \\
\hline $\begin{array}{l}\text { Jagged1 loss of function } \\
\text { Jagged1/Notch1 loss } \\
\text { of function }\end{array}$ & Mx-Cre & Mouse & $\begin{array}{l}\text { No effect on progenitor nor } \\
\text { mature lymphoid, myeloid, } \\
\text { and erythroid lineages }\end{array}$ & $\begin{array}{l}\text { Normal LSK function } \\
\text { and numbers }\end{array}$ & {$[22]$} \\
\hline RBPJk loss of function & Mx-Cre & Mouse & $\begin{array}{l}\text { Impaired T cell development; } \\
\text { myeloid and B cell } \\
\text { development is normal }\end{array}$ & $\begin{array}{l}\text { Normal HSC function } \\
\text { and numbers }\end{array}$ & {$[23]$} \\
\hline Notch1 and 2 loss of function & Mx-Cre & Mouse & $\begin{array}{l}\text { Myeloid and B cell } \\
\text { development is normal } \\
\text { in steady-state }\end{array}$ & $\begin{array}{l}\text { Normal HSC function } \\
\text { and numbers }\end{array}$ & {$[24]$} \\
\hline dnMAML1 transduced HSCs & $\begin{array}{l}\text { Transduction CB cells } \\
\text { with dnMALM1 }\end{array}$ & Human & $\begin{array}{l}\text { Impaired T cell development; } \\
\text { myeloid and B cell } \\
\text { development is normal }\end{array}$ & $\begin{array}{l}\text { Reduction in frequency } \\
\text { but increase in HSC } \\
\text { numbers }\end{array}$ & {$[25 \bullet \bullet]$} \\
\hline $\begin{array}{l}\text { Notch inhibition } \\
\quad(\gamma \text {-secretase inhibitor })\end{array}$ & $\begin{array}{l}\text { DAPT ( } \gamma \text {-secretase } \\
\text { inhibitor) administration } \\
\text { in vivo }\end{array}$ & Human & Early differentiation of HSPCs & $\begin{array}{l}\text { Higher engraftment capacity } \\
\text { (increased generation of } \\
\text { CD34+CD38- from } \\
\text { CD34- HSPCs) }\end{array}$ & {$[26 \bullet \bullet]$} \\
\hline
\end{tabular}

$N D$ not determined

proposed that LSK cells are exposed to very low levels of Notch signaling in vivo, contrasting to the observations reported by Duncan et al. This could explain the reason why the loss of canonical Notch does not result in dramatic effects in vivo and could also justify why in different studies, exposing cells to super-physiological levels of ligands or NICD in vitro resulted in significant increase in stem cell selfrenewal.

Beyond the divergent data in mice, only recently some light on the role of Notch pathway in human HSCs in vivo has been shed. Benveniste et al. directly compared the impact of canonical Notch pathway on human purified HSCs in vitro and in vivo using the same strategy in both contexts [25••]. By overexpressing dnMAML1, the authors showed that the contribution to all the hematopoietic lineages in vivo in a xenogeneic model was comparable in a competition assay between control- and dnMAML1-HSCs, despite complete blockage of T-lineage development. Differently, the overexpression of the dnMAML1 blocked both T cell development and HSC maintenance/expansion in vitro. Although the authors appear to claim similar outcomes to the murine system [23], a close inspection of the authors' results could infer a different interpretation. First, the percentage of dnMAML1-HSC population within the human graft was half as compared to controls. Second, the total number of dnMAML1 engrafted cells was more than double compared to controls; hence, the authors concluded that the total number of engrafted dnMAML1HSC was unchanged relative to control HSCs [25••]. However, it can be inferred that it did as the frequency of dnMAML1-HSC was reduced with concomitant increase in total differentiated human cells.

Furthermore, the authors did not consider the possibility of non-canonical functions of the Notch receptors as a compensatory mechanism. Indeed, Delta-like1 ligand can rewire interleukin-6-mediated signal in human CD34 $4^{+}$cells through as yet unknown non-canonical mechanisms [28]. Also, they did not study the Notch pathway in human CD34- HSCs or consider potential non-cell-autonomous effects that could play a role when Notch signaling is not only inhibited in HSCs. Indeed, at the top of the human hematopoietic hierarchy resides a quiescent and immature population, defined as $\mathrm{Lin}^{-} \mathrm{CD} 34^{-} \mathrm{CD} 38^{-} \mathrm{CD} 93^{\text {hi }}(-/-/+)$, that has higher Notch activity as compared to the more mature but still enriched $\mathrm{CD} 34^{+}$ HSCs population $\left(\mathrm{Lin}^{-} \mathrm{CD} 34^{+} \mathrm{CD} 45 \mathrm{RA}^{-} \mathrm{CD} 90^{+} \mathrm{CD} 49 \mathrm{f}^{+}\right.$). The engraftment potential of the $-/-/+$ HSCs increased after administration of the $\gamma$-secretase inhibitor DAPT (a Notch pathway inhibitor) to the murine host in a xenograft model [26••]. Although more prolonged DAPT administration further increased human engraftment, this occurred at the expense of the $\mathrm{CD} 34^{+}$cells that are normally derived from the $-/-1+$ HSCs. These results suggest that the activation of the Notch pathway is important for the maintenance of the human HSCs 
repopulation capacity. However, the potential effects induced by the microenvironment were not determined since DAPT treatment may have also affected the niche cells.

In summary, these discrepancies underline the necessity for having more stringent and well-described methods to disrupt Notch signaling in vivo, but they also highlight a potential difference between the murine and the human systems.

\section{Loss of Notch Signaling and Myeloproliferative Disease}

In contrast to the above results, several studies using mouse models, in which different members of the Notch signaling pathway have been deleted, have demonstrated that defective Notch in the bone marrow microenvironment frequently leads to the development of a myeloproliferative disease (MPD) phenotype (Table 2).

One of the earliest reports has shown that Presenilin1 haplo- and Presenilin2-deficient $\left(P \operatorname{sen} 1^{+-} \operatorname{Psen}^{-/-}\right)$mice have an expanded granulocytic compartment, and the animals develop signs of MPD [29]. Notch receptors are subjected to a great amount of post-translational modifications. Of those, Ofucose residues added to the receptors by the FX (Factor 10; homolog of human GDP-L-fucose synthase) enzyme are a substrate for further modification by Fringe proteins. FX mediates the addition of $\mathrm{N}$-acetylglucosamine residues [36], which are essential for proper interaction with ligands [37]. $\mathrm{Fx}^{-/-}$mice develop a fucosylation-dependent myeloproliferative phenotype, with expanded $\mathrm{Gr}^{+}$granulocytes and Ter $119^{+}$erythrocytes [31]. Additionally, these mutant mice have increased common myeloid progenitor (CMP), granulocyte-macrophage progenitor (GMP), and megakaryocyte-erythrocyte progenitor (MEP) compartments, while the common lymphoid progenitors (CLPs) are significantly reduced [31]. Similarly to FX-deficient cells, Pofut (protein O-fucosyltransferase)-deficient marrow progenitors have defective O-fucosylation of Notch receptors and have no ability to bind to Delta-like ligands. Pofut-deficient mice have increased numbers of neutrophils and reduced lymphocytes. However, retrovirally transducing Pofut-deficient cells with N1-ICD can rescue the T cell development impairment and the $\mathrm{Gr}^{+}$cell expansion, suggesting a role for O-fucosedependent Notch1 activity [32]. Interestingly, these observations suggest that Notch must be important at an earlier stage, perhaps at the multipotent progenitor stage, where the finetuning of the pathway regulates the lymphoid versus myeloid cell fate decisions.

In addition, using two Cre lines, MMTV-Cre and Mx1-Cre, Kim and colleagues showed that the loss of Mindbomb-1 (Mib1), an essential component for Notch ligands endocytosis, led to MPD originated from the LSK population [30]. However, transplantation of bone marrow cells from TNR reporter mice showed that LSKs from reconstituted Mib1mutant mice had the same level of Notch activation as wildtype cells, showing that the MPD is not caused by defective Notch receptor cleavage in LSK cells. In fact, the constitutive expression of N1-ICD in Mib1-null microenvironment significantly delayed the development of MPD, suggesting that the defective Notch signaling between the microenvironment cells caused the MPD phenotype. Nevertheless, this data suggests that appropriate Notch signaling in the niche cells has an impact on LSK biology.

Another example relates to Adam 10 deletion in the bone marrow compartment, which leads to both cell-autonomous and non-cell-autonomous MPD. In both cases, deregulated Notch pathway triggers granulopoiesis with an expanded HSC pool [33]. These results suggest that proper Notch signaling must be maintained not only in HSPCs but also in the bone marrow microenvironment. This entails that Notch signaling is most likely important in the crosstalk between hematopoietic and niche cells, whereby the loss of proper Notch signaling in non-hematopoietic cells results in deregulated stem and progenitor cell self-renewal and differentiation. An interesting example is a skin-specific inactivation of Notchl and Notch2, or Rbpj, which results in a myeloproliferative disorder with increased immature myeloid cells in the bone marrow and spleen. This was caused by non-cell autonomous
Table 2 Notch signaling and MPD: involvement of the microenvironment

\begin{tabular}{|c|c|c|c|}
\hline Mouse model & Phenotype & Effect on stem cells & Reference \\
\hline Psen $1^{+/-} / \operatorname{Psen} 2^{-/-}$ & MPD (expanded granulocytes) & Normal side population & [29] \\
\hline MMTV-Cre/Mib1 ${ }^{\mathrm{f} / \mathrm{f}}$ & MPD (expanded granulocytes) & Expanded LSK & {$[30]$} \\
\hline $\begin{array}{l}\mathrm{Mx} 1-\mathrm{Cre} / \mathrm{Mib}^{\mathrm{f} / \mathrm{f}} \\
\mathrm{Fx}^{-/-}\end{array}$ & MPD & Normal LSK & {$[31]$} \\
\hline Mx1-Cre/Pofut ${ }^{\mathrm{f} / \mathrm{f}}$ & Increased neutrophils & ND & {$[32]$} \\
\hline Mx1-Cre/Adam $10^{\mathrm{f} / \mathrm{f}}$ & MPD (expanded) & Expanded LSK & {$[33]$} \\
\hline $\mathrm{Mx} 1-\mathrm{Cre} / \mathrm{Ncstn}{ }^{\mathrm{f} / \mathrm{f}}$ & CMML-like & Expanded LSK & {$[34]$} \\
\hline $\begin{array}{l}\text { Mx1-Cre/Rbpj }{ }^{\mathrm{f} / \mathrm{f}} \\
\mathrm{Tie}-\mathrm{Cre} / \mathrm{Rbpj}{ }^{\mathrm{f} / \mathrm{f}}\end{array}$ & MPD & Expanded LSK & {$[35 \bullet \bullet]$} \\
\hline
\end{tabular}

$N D$ not determined 
microenvironmental changes in which the loss of Notch triggered the production of thymic stromal lymphopoietin that led to increased levels of G-CSF resulting in non-cellautonomous MPD [38].

Subsequently, an important work from Aifantis's group revealed that mice transplanted with Nicastrin-deficient HSCs develop a human chronic myelomonocytic leukemialike disease with an expanded LSK compartment. This is one of the few studies that attempted to understand the mechanisms behind this effect, with the demonstration that Nicastrin-deficient LSK cells showed a derepressed myeloid program, which is thought to be carried out by HES1mediated inhibition of Cebpa and Spil expressions [34].

Finally, an elegant study from the Carlesso laboratory showed that conditional deletion of Rbpj using Mx1-Cre leads to myeloproliferative disease [35*0]. When performing reciprocal transplantation experiments, the authors observed that the mutant-LSK pool was significantly increased but the GMP, CMP, and MEP subsets were unaffected in wild-type mice but without signs of splenomegaly and with similar survival as the control cohort. In contrast, when transplanting wild-type HSPCs into mutant mice, CMPs and GMPs in the bone marrow and spleen were significantly increased similarly to the parental $\mathrm{Rbpj}^{--}$mice, resulting in lethal MPD. Interestingly, the disease evolved faster after bone marrow transplant. Additionally, the wild-type LSK pool in $\mathrm{Rbpj}^{-/}$ recipients was less affected than in parental $R b p j^{-1-}$ mice or $\mathrm{Rbpj}^{-/-}$cells transplanted into wild-type mice demonstrating a cell-autonomous contribution of RBPJ in HSC regulation. However, this study also demonstrates that cell autonomous loss of Notch activation is not sufficient to develop myeloid disease, while loss of Notch signaling in the microenvironment induces lethal MPD in a non-cell-autonomous manner. Mechanistically, deletion of Rbpj leads to increased expression of miR-155 in bone marrow niche cells where the NICD/ RBPJ complex acts as a transcription repressor of miR-155. miR-155 upregulates NF- $\mathrm{kB}$ activation by targeting one of its inhibitors $\mathrm{KB}$-Ras 1, leading to increased expression of G-CSF and $\mathrm{TNF} \alpha$ thus, inducing a persistent inflammatory state in the bone marrow.

In summary, it is remarkable that inhibition of Notch, through the loss of different regulators of the pathway, seems to expand the most primitive HSCs. This is probably due to early differentiation induced by the loss of a quiescent state imposed by Notch signaling. It will be important to clarify whether this effect leads to stem cell exhaustion or if in some cases, stem cells can divide seemingly indefinitely as has been described before [18]. But most importantly, these studies demonstrate that Notch activation via Notch ligand-receptor interactions between niche cells is also required for normal hematopoiesis. Altogether, these studies highlight the importance of Notch signaling in the bone marrow microenvironment for the proper regulation of hematopoiesis.

\section{HSC and Niche Crosstalk Via Notch Signaling}

Several studies have looked for the presence and functions of Notch ligands in different bone marrow niche cells (endothelial cells, osteoblasts, and mesenchymal stromal cells) that are able to support HSPCs in vitro or appear closely associated to them in vivo.

Indeed, bone marrow endothelial cells express Notch ligands and its expression can be regulated by proinflammatory stimuli in vitro and in vivo [39]. In a study by Fernandez and colleagues, a human bone marrow endothelial cell line called "BMEC" and primary human bone marrow endothelial cells were found to express Jagged ligands. The capacity of these cells to expand hematopoietic progenitors in an in vitro co-culture system was dependent on Notch signaling. The authors also demonstrated that the ligand density and Notch signal intensity could result in different degrees of HSPCs expansion. Furthermore, in vivo pro-inflammatory cues (TNF $\alpha$, LPS) caused an increase of Jagged2 expression on endothelial cells and Notch1 and Notch2 receptors on hematopoietic progenitors, which were associated with increased Notch activation.

Later, Butler and colleagues showed that endothelial cells are able to support long-term expansion of Notch-activated $\left(\mathrm{TNR}^{-G F P}{ }^{+}\right.$) LSK HSPCs, but not Notch1 and Notch2 defective HSCs (Notch $1^{-/}$Notch $2^{-/}$CD $34^{-}$Flt- $3^{-}$LSK) [40]. The authors also elegantly showed that HSCs secrete vascular endothelial growth factor A (VEGF-A) when exposed to soluble stem cell factor in vitro. VEGF-A secreted by HSCs in turn stimulates the translocation of Jagged2 ligand on the endothelial cell surface, which then supports the expansion of the HSCs by interacting with Notch1 and Notch2 receptors. This study also demonstrated that in vivo inhibition of both VEGFR2 and VE-cadherin, essential for endothelial angiocrine signaling, resulted in a profound decrease in the instructive capacity of endothelial cells. This caused impaired reconstitution of TNR-GFP ${ }^{+}$hematopoietic cells after sublethal irradiation due to a significant decrease in the regeneration of TNR-GFP ${ }^{+} \mathrm{HSCs}$, leading to demise of the treated mice. These results not only support the importance of the vascular niche but also suggest that during hematopoietic recovery, angiocrine expression of Notch ligands by endothelial cells is essential to balance the expansion and differentiation of HSCs and also reveal a crosstalk between HSCs and their niche elements.

In the same vein, Butler's group showed that in vivo conditional deletion of Jagged1 in endothelial cells (VE-cadherinCre) caused a decrease in the number of phenotypical and functional HSCs in steady-state, which was associated with a significant reduction in Notch activation in LSK HSPCs [41•]. In addition, mutant mice have deficient hematopoietic recovery after sub-lethal irradiation leading to reduced survival rate compared to the control group. In vitro co-cultures with 
endothelial cells from mutant mice resulted in greater hematopoietic expansion exclusively from differentiated cells with a significant increase in myeloid cells. These data emphasize the importance of niche Jagged ligands in balancing expansion and differentiation of HSPCs, demonstrating that Notch signaling activation by bone marrow endothelial cells through Jagged1 ligand is essential to maintain homeostatic and regenerative hematopoiesis.

Of note, this data contradicts the observations of Mancini et al. [22], in which conditional deletion of Jagged 1 driven by the Mx1 promoter had no effect on hematopoietic regulation at steady-state or regenerative conditions. Butler's group verified that while the VE-cadherin-Cre efficiently deleted the floxed Jaggedl in bone marrow endothelial cells, the inducible Mx1-Cre system did not. These data indicate that the observations of Mancini et al. could be associated to inefficient deletion of endothelial-specific Jagged1.

Apart from endothelial cells, other bone marrow stromal cells have also been shown to be associated to HSPC regulation via Notch signaling. A subset of human CD146 perivascular mesenchymal stromal cells (MSCs) support long-term culture of functional human HSPCs partially through Notch activation [42]. Indeed, CD146 ${ }^{+}$perivascular cells express high levels of Notch ligand Jagged1. Accordingly, Notch activation was demonstrated to be significantly higher in HSPCs co-cultured with $\mathrm{CD} 146^{+}$cells than in co-cultures with total MSCs or CD146 cells. Moreover, Notch inhibition with DAPT resulted in decreased HSPCs number and CFUs and increased B cell differentiation. These results suggest that $\mathrm{CD}_{146^{+}}$perivascular cells can also regulate self-renewal and lineage commitment of HSPCs through Notch signaling.

Interestingly, a recent study shed some light on the human bone marrow niche in vivo by analyzing human bone biopsy specimens and human-mouse xenografts. This study revealed that HSCs with superior regenerative and self-renewal capacity tend to localize to endosteal regions of the trabecular bone area (TBA) [27••]. These HSCs have distinct molecular activation enriched with Notch signaling signature compared to HSCs localizing in the long bone area (LBA). Particularly within the TBA, phenotypic human HSCs and HPCs preferentially locate in the endosteal over vascular regions. The authors also showed that osteoblasts from the TBA have increased expression of Notch ligands Jagged1, Jagged2, and Delta-like4 compared to osteoblasts from the LBA, consistent with the HSC activation profile. In particular, a threefold higher proportion of osteoblasts expressing Jagged1 was detected in the TBA compared to LBA. Additionally, Jagged1binding HSPCs were found to have higher CFU capacity and repopulating capacity. Upon Notch inhibition with DAPT treatment in vivo, total human engraftment and HSPC numbers were not affected in the LBA, whereas there was a significant reduction in the TBA, suggesting that only this region is sensitive to Notch inhibition leading to an overall reduction in human engraftment in a region-specific manner. These observations support a critical role of Notch signaling in HSC regulation through interactions with specific bone marrow niche cells.

In a subsequent study, further investigation on the role of endosteal osteoblasts in HSC regulation showed that osteoblast ablation (Col2.3 $\Delta$ tk) leads to reduced quiescence, long-term engraftment, and self-renewal capacity of HSCs [43]. Moreover, osteoblast ablation in a transgenic chronic myeloid leukemia mouse model showed accelerated leukemia progression with reduced survival compared to control mice. In this mouse model, osteoblasts were found to express high levels of Jagged 1 and co-culture of leukemic or normal HSCs with stromal cells expressing Jagged1 resulted in reduced cell cycling and total number of cells. These data suggest that Jagged1 signaling in the osteogenic niche is essential for maintaining HSC quiescence and long-term self-renewal capacity.

Finally, as further evidence of the importance of Notch ligands in the osteogenic niche, in humanized mice expressing human Jagged1 in osteoblasts, human HSPC repopulation is higher than in control mice [44]. In addition, mice with human Delta-like1-expressing osteoblasts develop severe osteosclerosis, which suppresses the generation of human B lymphocytes and also retention of human HSPCs that is likely due to the severe osteosclerosis in the bone marrow [45].

Together, these studies focusing on the osteogenic niche suggest a role for Notch signaling through osteoblasts on regulation of HSC self-renewal and quiescence, particularly through Jagged1 in the trabecular bone area, where interactions and Notch activation seem to be higher. However, the activated Notch receptors and the molecular mechanisms behind this regulation are still not understood. Also, a more comprehensive study is necessary to clearly identify the functions of the different ligands on HSPC regulation both in vitro and in vivo.

\section{Conclusions}

Although several authors have suggested Notch signaling as dispensable in the adult hematopoietic pathway using different genetic approaches, the maintenance of adequate dose of Notch signaling seems fundamental in order to avoid the development of hematological malignancies. As reported, different populations within the primitive hematopoietic stem and progenitor compartments seem to depend differently on Notch at different stages of differentiation. As already mentioned, it will be relevant to understand the functions of the different ligand/receptor pairings in different primitive populations. We have reviewed some studies that have shed light on the mechanisms by which Notch regulate HSPCs, and will certainly 
learn more about the fine-tuning of this signaling pathway as soon as more stringent and well-described methods to inhibit and activate Notch become available.

\section{Compliance with Ethical Standards}

Conflict of Interest Fabio Pereira Lampreia, Joana Gonçalves Carmelo, and Fernando Anjos-Afonso declare that they have no conflict of interest.

Human and Animal Rights and Informed Consent All reported studies/experiments with human or animal subjects performed by the authors have been previously published and complied with all applicable ethical standards (including the Helsinki Declaration and its amendments, institutional/national research committee standards, and international/national/institutional guidelines).

Open Access This article is distributed under the terms of the Creative Commons Attribution 4.0 International License (http:// creativecommons.org/licenses/by/4.0/), which permits unrestricted use, distribution, and reproduction in any medium, provided you give appropriate credit to the original author(s) and the source, provide a link to the Creative Commons license, and indicate if changes were made.

\section{References}

Papers of particular interest, published recently, have been highlighted as:

- Of importance

•- Of major importance

1. Bray SJ. Notch signalling: a simple pathway becomes complex. Nat Rev Mol Cell Biol. 2006;7(9):678-89. doi:10.1038/nrm2009.

2. Andersson ER, Sandberg R, Lendahl U. Notch signaling: simplicity in design, versatility in function. Development. 2011;138(17): 3593-612. doi:10.1242/dev.063610.

3. Iso T, Kedes L, Hamamori Y. HES and HERP families: multiple effectors of the Notch signaling pathway. J Cell Physiol. 2003;194(3):237-55. doi:10.1002/jcp.10208.

4. Andersen P, Uosaki H, Shenje LT, Kwon C. Non-canonical Notch signaling: emerging role and mechanism. Trends Cell Biol. 2012;22(5):257-65. doi:10.1016/j.tcb.2012.02.003.

5. Artavanis-Tsakonas S, Rand MD, Lake RJ. Notch signaling: cell fate control and signal integration in development. Science. 1999;284(5415):770-6.

6. Greenwald IS, Sternberg PW, Horvitz HR. The lin-12 locus specifies cell fates in Caenorhabditis elegans. Cell. 1983;34(2):435-44.

7. Ellisen LW, Bird J, West DC, Soreng AL, Reynolds TC, Smith SD, et al. TAN-1, the human homolog of the Drosophila notch gene, is broken by chromosomal translocations in T lymphoblastic neoplasms. Cell. 1991;66(4):649-61.

8. Weng AP, Ferrando AA, Lee W, Morris JP, Silverman LB, SanchezIrizarry $\mathrm{C}$, et al. Activating mutations of NOTCH1 in human T cell acute lymphoblastic leukemia. Science. 2004;306(5694):269-71. doi:10.1126/science. 1102160

9. Kumano K, Chiba S, Kunisato A, Sata M, Saito T, NakagamiYamaguchi E, et al. Notch1 but not Notch2 is essential for generating hematopoietic stem cells from endothelial cells. Immunity. 2003;18(5):699-711.

10. Radtke F, Wilson A, Stark G, Bauer M, van Meerwijk J, MacDonald HR, et al. Deficient $\mathrm{T}$ cell fate specification in mice with an induced inactivation of Notch1. Immunity. 1999;10(5): $547-58$.

11. Calvi LM, Adams GB, Weibrecht KW, Weber JM, Olson DP, Knight MC, et al. Osteoblastic cells regulate the haematopoietic stem cell niche. Nature. 2003;425(6960):841-6. doi:10.1038/ nature 02040.

12. Duncan AW, Rattis FM, DiMascio LN, Congdon KL, Pazianos G, Zhao C, et al. Integration of Notch and Wnt signaling in hematopoietic stem cell maintenance. Nat Immunol. 2005;6(3):314-22. doi:10.1038/ni1164.

13. Milner LA, Kopan R, Martin DI, Bernstein ID. A human homologue of the Drosophila developmental gene, Notch, is expressed in CD34+ hematopoietic precursors. Blood. 1994;83(8):2057-62.

14. Carlesso N, Aster JC, Sklar J, Scadden DT. Notch1-induced delay of human hematopoietic progenitor cell differentiation is associated with altered cell cycle kinetics. Blood. 1999;93(3):838-48.

15. Kunisato A, Chiba S, Nakagami-Yamaguchi E, Kumano K, Saito T, et al. HES-1 preserves purified hematopoietic stem cells ex vivo and accumulates side population cells in vivo. Blood. 2003;101(5): 1777-83. doi:10.1182/blood-2002-07-2051.

16. Varnum-Finney B, Brashem-Stein C, Bernstein ID. Combined effects of Notch signaling and cytokines induce a multiple log increase in precursors with lymphoid and myeloid reconstituting ability. Blood. 2003;101(5):1784-9. doi:10.1182/blood-2002-06-1862.

17. Karanu FN, Murdoch B, Gallacher L, Wu DM, Koremoto M, Sakano S, et al. The notch ligand jagged-1 represents a novel growth factor of human hematopoietic stem cells. J Exp Med. 2000;192(9):1365-72.

18. Rathinam C, Matesic LE, Flavell RA. The E3 ligase Itch is a negative regulator of the homeostasis and function of hematopoietic stem cells. Nat Immunol. 2011;12(5):399-407. doi:10.1038/ni. 2021.

19. Ohishi K, Varnum-Finney B, Bernstein ID. Delta-1 enhances marrow and thymus repopulating ability of human CD34(+)CD38(-) cord blood cells. J Clin Invest. 2002;110(8):1165-74. doi:10.1172/ JCI16167.

20. Lauret E, Catelain C, Titeux M, Poirault S, Dando JS, Dorsch M, et al. Membrane-bound delta-4 notch ligand reduces the proliferative activity of primitive human hematopoietic CD34+CD38low cells while maintaining their LTC-IC potential. Leukemia. 2004;18(4):788-97. doi:10.1038/sj.leu.2403288.

21. Han H, Tanigaki K, Yamamoto N, Kuroda K, Yoshimoto M, Nakahata $\mathrm{T}$, et al. Inducible gene knockout of transcription factor recombination signal binding protein-J reveals its essential role in $\mathrm{T}$ versus B lineage decision. Int Immunol. 2002;14(6):637-45.

22. Mancini SJ, Mantei N, Dumortier A, Suter U, MacDonald HR, Radtke F. Jagged1-dependent Notch signaling is dispensable for hematopoietic stem cell self-renewal and differentiation. Blood. 2005;105(6):2340-2. doi:10.1182/blood-2004-08-3207.

23. Maillard I, Koch U, Dumortier A, Shestova O, Xu L, Sai H, et al. Canonical notch signaling is dispensable for the maintenance of adult hematopoietic stem cells. Cell Stem Cell. 2008;2(4):356-66. doi:10.1016/j.stem.2008.02.011.

24. Varnum-Finney B, Halasz LM, Sun M, Gridley T, Radtke F, Bernstein ID. Notch2 governs the rate of generation of mouse long- and short-term repopulating stem cells. J Clin Invest. 2011;121(3):1207-16. doi:10.1172/JCI43868.

25.• Benveniste P, Serra P, Dervovic D, Herer E, Knowles G, Mohtashami M, et al. Notch signals are required for in vitro but not in vivo maintenance of human hematopoietic stem cells and delay the appearance of multipotent progenitors. Blood. 2014;123(8):1167-77. doi:10.1182/blood-2013-07-505099. Uses dnMAML1-transduced human HSPCs to suggest different requirements for Notch signaling in vitro and in vivo

26.• Anjos-Afonso F, Currie E, Palmer HG, Foster KE, Taussig DC, Bonnet D. CD34(-) cells at the apex of the human hematopoietic 
stem cell hierarchy have distinctive cellular and molecular signatures. Cell Stem Cell. 2013;13(2):161-74. doi:10.1016/j.stem.2013.05.025. This study shows that a rare $\mathrm{Lin}^{-} \mathrm{CD} 34^{-} \mathrm{CD}^{-} 8^{-} \mathrm{CD} 93^{\text {hi }}$ population has active Notch signaling, whereby Delta4 and Jagged1 are crucial for the maintenance of a quiescent state

27.• Guezguez B, Campbell CJ, Boyd AL, Karanu F, Casado FL, Di Cresce $\mathrm{C}$, et al. Regional localization within the bone marrow influences the functional capacity of human HSCs. Cell Stem Cell. 2013;13(2):175-89. doi:10.1016/j.stem.2013.06.015. This report demonstrates that HSCs in trabecular bone area are enriched in Notch signaling and show superior regenerative and selfrenewal capacity compared to HSCs from long bone area

28. Csaszar E, Wang W, Usenko T, Qiao W, Delaney C, Bernstein ID, et al. Blood stem cell fate regulation by Delta-1-mediated rewiring of IL-6 paracrine signaling. Blood. 2014;123(5):650-8. doi:10. 1182/blood-2013-08-520445.

29. Qyang Y, Chambers SM, Wang P, Xia X, Chen X, Goodell MA, et al. Myeloproliferative disease in mice with reduced presenilin gene dosage: effect of gamma-secretase blockage. Biochemistry. 2004;43(18):5352-9. doi:10.1021/bi049826u.

30. Kim YW, Koo BK, Jeong HW, Yoon MJ, Song R, Shin J, et al. Defective Notch activation in microenvironment leads to myeloproliferative disease. Blood. 2008;112(12):4628-38. doi:10.1182/ blood-2008-03-148999.

31. Zhou L, Li LW, Yan Q, Petryniak B, Man Y, Su C, et al. Notchdependent control of myelopoiesis is regulated by fucosylation. Blood. 2008;112(2):308-19. doi:10.1182/blood-2007-11-115204.

32. Yao D, Huang Y, Huang X, Wang W, Yan Q, Wei L, et al. Protein O-fucosyltransferase 1 (Pofut1) regulates lymphoid and myeloid homeostasis through modulation of Notch receptor ligand interactions. Blood. 2011;117(21):5652-62. doi:10.1182/blood-2010-12326074.

33. Yoda M, Kimura T, Tohmonda T, Uchikawa S, Koba T, Takito J, et al. Dual functions of cell-autonomous and non-cell-autonomous ADAM10 activity in granulopoiesis. Blood. 2011;118(26):693942. doi:10.1182/blood-2011-06-357210.

34. Klinakis A, Lobry C, Abdel-Wahab O, Oh P, Haeno H, Buonamici $\mathrm{S}$, et al. A novel tumour-suppressor function for the Notch pathway in myeloid leukaemia. Nature. 2011;473(7346):230-3. doi:10. 1038/nature09999.

35.• Wang L, Zhang H, Rodriguez S, Cao L, Parish J, Mumaw C, et al. Notch-dependent repression of miR-155 in the bone marrow niche regulates hematopoiesis in an NF-kB-dependent manner. Cell Stem Cell. 2014;15(1):51-65. doi:10.1016/j.stem.2014.04.021. This study explores a non-canonical aspect of Notch signaling whereby loss of Notch upregulates miR-155 resulting in myeloproliferative disease

36. Becker DJ, Lowe JB. Fucose: biosynthesis and biological function in mammals. Glycobiology. 2003;13(7):41R-53R. doi:10.1093/ glycob/cwg054.

37. Okajima $\mathrm{T}, \mathrm{Xu} \mathrm{A}$, Irvine $\mathrm{KD}$. Modulation of notch-ligand binding by protein $\mathrm{O}$-fucosyltransferase 1 and fringe. $\mathrm{J}$ Biol Chem. 2003;278(43):42340-5. doi:10.1074/jbc.M308687200.

38. Dumortier A, Durham AD, Di Piazza M, Vauclair S, Koch U, Ferrand G, et al. Atopic dermatitis-like disease and associated lethal myeloproliferative disorder arise from loss of Notch signaling in the murine skin. PLoS One. 2010;5(2):e9258. doi:10.1371/journal. pone.0009258.

39. Fernandez L, Rodriguez S, Huang H, Chora A, Fernandes J, Mumaw C, et al. Tumor necrosis factor-alpha and endothelial cells modulate Notch signaling in the bone marrow microenvironment during inflammation. Exp Hematol. 2008;36(5):545-58. doi:10. 1016/j.exphem.2007.12.012.

40. Butler JM, Nolan DJ, Vertes EL, Varnum-Finney B, Kobayashi H, Hooper AT, et al. Endothelial cells are essential for the self-renewal and repopulation of Notch-dependent hematopoietic stem cells. Cell Stem Cell. 2010;6(3):251-64. doi:10.1016/j.stem.2010.02.001.

41. Poulos MG, Guo P, Kofler NM, Pinho S, Gutkin MC, Tikhonova A, et al. Endothelial Jagged-1 is necessary for homeostatic and regenerative hematopoiesis. Cell Rep. 2013;4(5):1022-34. doi:10. 1016/j.celrep.2013.07.048. Conditional deletion of Jagged1 in bone marrow endothelial cells demonstrated the importance of Notch signaling in balancing expansion and differentiation of HSPCs in homeostatic and regenerative conditions

42. Corselli M, Chin CJ, Parekh C, Sahaghian A, Wang W, Ge S, et al. Perivascular support of human hematopoietic stem/progenitor cells. Blood. 2013;121(15):2891-901. doi:10.1182/blood-2012-08451864.

43. Bowers M, Zhang B, Ho Y, Agarwal P, Chen CC, Bhatia R. Osteoblast ablation reduces normal long-term hematopoietic stem cell self-renewal but accelerates leukemia development. Blood. 2015;125(17):2678-88. doi:10.1182/blood-2014-06-582924.

44. Negishi N, Suzuki D, Ito R, Irie N, Matsuo K, Yahata T et al. Effective expansion of engrafted human hematopoietic stem cells in bone marrow of mice expressing human Jagged1. Exp Hematol 2014;42(6):487-494 e1. doi:10.1016/j.exphem.2014.02.001.

45. Ito R, Negishi N, Irie N, Matsuo K, Suzuki D, Katano I et al. Osteosclerosis and inhibition of human hematopoiesis in NOG mice expressing human Delta-like 1 in osteoblasts. Exp Hematol 2012;40(11):953-963 e3. doi:10.1016/j.exphem.2012.07.002. 